\title{
The interdependence of time and space in somesthesis: The Tau effect reexamined
}

\author{
EUGENE C. LECHELT and RICHARD BORCHERT \\ University of Alberta, Edmonton, Alberta, Canada T6G $2 E 9$
}

\begin{abstract}
The Tau effect, whereby the comparative perception of two spaces is altered by the relationship of the temporal intervals associated with them, was examined in the tactile sense. A bilateral stimulus presentation procedure was employed in which the standard and comparison spatial separations were presented alternately to either forearm. The temporal intervals separating the two stimuli delimiting the standard and comparison spatial separations were manipulated over a wide range. Subjects' judgments of the comparison spatial separation, relative to the standard separation, were altered substantially by varying the temporal relations between the standard and comparison spaces, that is, the Tau effect was obtained. No systematic or substantial laterality effect was found, that is, varying the position of the standard and comparison spaces on the right or left forearm had only a negligible effect.
\end{abstract}

Manipulations in space and time provide the major variations in experimental conditions in investigations of human perceptual discrimination. Research has shown that spatial and temporal components of space-time events about which judgments are made are psychologically interdependent. The most direct experimental verification of the interdependence of time and space as a primary feature of experience has come from studies of comparative judgments of either spatial extent or temporal interval between successive stimulations. Judgments of temporal intervals between successively presented stimuli have been shown to be dependent upon the spatial relations between the stimuli; specifically, the temporal interval between two successive stimuli is perceived to lengthen as the spatial distance between the corresponding stimuli increases. This phenomenon has been called the Kappa effect and has been found in vision (Cohen, Hansel, \& Sylvester, 1953; Price-Williams, 1954), audition (Cohen, Hansel, \& Sylvester, 1954, 1955), and has been studied in the tactile sense (Yoblick \& Salvendy, 1970). Conversely, judgments of the spatial extent between two stimuli also have been reported to be dependent upon the temporal interval between the stimuli; specifically, a space defined by a short interval of time between two successive stimulations appears shorter than a physically identical extent defined by a longer interval. This phenomenon has been called the Tau effect and has been demonstrated in vision (Geldreich, 1934), somesthesis (Helson \& King, 1931), and audition (Cohen, Hansel, \& Sylvester, 1954, 1955).

Only one study has quantitatively specified the Tau effect in the tactile modality (Helson \& King, 1931).

This research was supported by Grant A8621 from the National Research Council of Canada to E. C. Lechelt.
The procedure used was to contact the right arm three places in temporal succession. The physical separation between Loci 1 and 2 as well as the temporal interval between their successive stimulation were always kept constant at $3 \mathrm{~cm}$ and $500 \mathrm{msec}$, respectively. However, the spatial separation between Loci 2 and 3 was varied by displacing the position of Locus 3 away from or toward Locus 2 in successive $5-\mathrm{cm}$ steps and the temporal interval between these loci was varied from 200 to $400 \mathrm{msec}$. Judgments of the second spatial extent were found to expand or contract in accordance with the temporal intervals between the stimulus "pairs." When the temporal intervals between stimulation of Loci 1 and 2 and 2 and 3 were equal, no spatial distortion occurred, but when the temporal intervals were imbalanced, subjects' judgments of the spatial separation between Loci 1 and 2 and 2 and 3 conformed to the temporal relations between 1 and 2 and 2 and 3 rather than to the spatial relations.

It would seem that two significant procedural aspects of the Helson and King (1931) experiment have not been, as yet, experimentally disentangled to establish their possible independent influence on the Tau effect. (1) The two spatial extents and temporal intervals shared a common locus; that is, the second stimulation locus was a "boundary" location for both standard and comparison spaces and simultaneously defined the end of the first interval and the onset of the second interval. (2) The total time of the two intervals shared by the successive "pairs" of stimulation was not kept constant or of equal duration.

The present experiment was designed to test the tactile Tau effect employing a bilateral stimulus presentation procedure. Such an experimental strategy represents not only important methodological improvements over previous research in this area, but also provides information of possible laterality differences 
in the magnitude of the Tau effect. Specifically, the two forearms were used as stimulation sites, with standard and comparison separations presented alternately to either arm in order to eliminate any possible confounding effect due to the standard and comparison separations sharing a common spatial and temporal locus as well as to specify any laterality effect. Also, although temporal imbalances between the successive stimulation of standard and comparison pairs were employed, the total time of the two intervals was kept constant so as not to permit differences in the total duration of the two temporal intervals to affect judgments of spatial extent.

\section{METHOD}

\section{Apparatus and Procedure}

The apparatus system included a stimulator control rack and an observer's support and stimulator table. The control rack housed a custom-designed automated stimulus generator and programmer which provided for precise control of stimulus intensity and interstimulus intervals. The tactile stimuli were 2-msec amplified rectangular mechanical pulses transmitted to a Bimorph transducer. The actual stimulating contactor on the skin surface was a $1 \frac{1 / 4}{4}$-in. $(.63-\mathrm{cm})$ diam Lucite rod rigidly affixed to and driven by the Bimorph transducer. On the stimulator table were armrests on which the observers appropriately positioned their right and left forearms. An elevated support system was attached to the table directly above the forearms. The transducers with affixed contactors were attached rigidly to precision dynamometers which were suspended from the support systems in a fashion enabling the experimenter to freely move them in any direction, so the contactors could be lowered on to the skin at specific locations and with specified amounts of static force.

A bilateral stimulation procedure was employed. Two contactors, the standard pair, were placed $10 \mathrm{~cm}$ apart along the center of the dorsal surface of one arm; another two contactors, the comparison pair, with variable spatial separations, were placed identically on the dorsal surface of the other arm. Each contactor was positioned at its appropriate spatial location on the skin to produce an initial static force of $15 \mathrm{~g}$. Distal placements of the contactors were the same for both arms, $3 \mathrm{~cm}$ proximal to the styloid process of the ulna. Proximal placement was $10 \mathrm{~cm}$ from the distal in the standard pair, but was varied in the comparison pair. During a preexperimental session, observers' arms were shaved to avoid complications arising from stimulating pressure spots by hairs, threshold for the tactile pulse was determined, and all loci were matched for sensory magnitudes. The absolute threshold for the $2-\mathrm{msec}$ pulse was determined for each observer at the distal locus on the right arm by the method of limits. The pulse intensity was then raised to $15 \mathrm{~dB}$ SL, and each locus was separately compared to the reference locus by successively raising and lowering the signal intensity at each comparison locus until the observer reported them to be of equivalent loudness. Great care was taken in balancing the loudness levels so that all loci were considered by each observer to be of equal subjective intensity. The observers had some familiarity with the procedure so threshold and balancing procedures were accomplished easily and with very little variation among individuals.

During experimental sessions, the four right-handed male subjects were seated comfortably at the stimulator table. The subjects were blindfolded throughout each session, and auditory cues during tactile stimulation were eliminated by continuously presenting $65 \mathrm{~dB}$ of white noise via stereo headphones. The method of constant stimulus differences was employed in a two-alternative forced-choice task. The standard spatial separation (S1) and comparison spatial separations (S2) were altered randomly between arms across experimental sessions but not within sessions. Similarly, although the two temporal separations between the two stimulus pairs always totaled $1,000 \mathrm{msec}$, for any given experimental session observers were assigned randomly to one of the five different ratios of t1 (interval associated with the standard spatial separation) to $t 2$ (interval associated with the comparison spatial separation). These ratios of $\mathrm{t} 1 / \mathrm{t} 2$ in milliseconds were: $800 / 200,650 / 350,500 / 500$, $350 / 650$, and $200 / 800$. The $t 1 / t 2$ ratio was varied across experimental sessions.

The procedure was self-paced in that observers initiated the onset of each trial by depressing a foot switch. A trial consisted of the presentation of the standard separation to one arm followed $1,500 \mathrm{msec}$ later by the comparison separation to the other arm. The subjects were instructed specifically to report immediately whether the second spatial separation was greater than or less than the standard. For each experimental session, seven comparison separations which fell within the observer's interval of uncertainty were presented randomly 10 times. The comparison separations differed in $.5-\mathrm{cm}$ steps so that the total extent of the variation remained constant at $3 \mathrm{~cm}$. Seventy reports were obtained from each observer in each of 10 experimental sessions-five $t 1 / t 2$ ratios by left or right forearm location of the standard.

\section{RESULTS AND DISCUSSION}

Previous research (Helson \& King, 1931) demonstrated the Tau effect under confounding stimulus

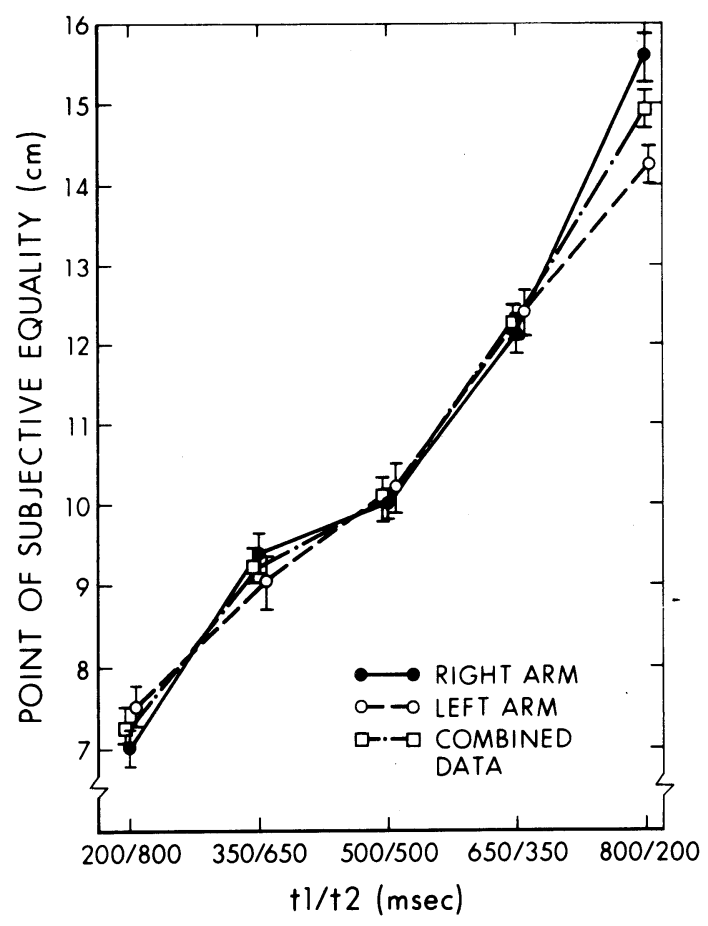

Figure 1. The mean points of subjective equality, that is, the value of the comparison spatial separation judged to be equal to the standard spatial separation of $10 \mathrm{~cm}$, as well as the standard deviations, averaged across subjects, are shown as a function of the temporal relations between the two temporal intervals when the standard spatial separation was on the right arm or left arm. Combined data, across arm conditions, are also shown. 
Table 1

Summary of the Temporal Manipulations, Mean PSEs for Comparison Spatial Separations, Temporal Interval and Spatial Separation Ratios, and Percentage of the Tau Effect

\begin{tabular}{|c|c|c|c|c|c|c|}
\hline $\begin{array}{c}1 \\
\mathrm{t} 1(\mathrm{msec})\end{array}$ & $\begin{array}{c}2 \\
\mathrm{t} 2(\mathrm{msec})\end{array}$ & $\begin{array}{c}3 \\
\text { S1 (cm) } \\
\text { (Standard) }\end{array}$ & $\begin{array}{c}4 \\
\mathrm{~S} 2(\mathrm{~cm}) \\
(\mathrm{PSE})\end{array}$ & $\begin{array}{c}5 \\
\mathrm{t} 1 / \mathrm{t} 2\end{array}$ & $\begin{array}{c}6 \\
\mathrm{~S} 2 / \mathrm{S} 1\end{array}$ & $\begin{array}{c}7 \\
(\mathrm{~S} 2-\mathrm{S} 1) / \mathrm{S} 1 \times 100\end{array}$ \\
\hline 800 & 200 & 10.0 & 14.9 & 4.00 & 1.49 & 49 \\
\hline 650 & 350 & 10.0 & 12.3 & 1.86 & 1.23 & 23 \\
\hline 500 & 500 & 10.0 & 10.1 & 1.00 & 1.01 & 1 \\
\hline 350 & 650 & 10.0 & 9.2 & .54 & .92 & -8 \\
\hline 200 & 800 & 10.0 & 7.3 & .25 & .73 & -27 \\
\hline
\end{tabular}

Note-Values of $t 1, t 2, S 1$ (standard spatial separation) and $S 2$ (mean comparison spatial separations averaged across subjects and arm conditions judged to be equal to S1) are shown in columns 1 through 4, respectively. In columns 5 and 6 the ratios of the temporal intervals and the reciprocal ratio of the spatial distances are shown as numbers, respectively. Column 7 gives the percentage of the Tau effect.

conditions in which standard and comparison spaces and times were part of a composite stimulus package. The present results provide clear support for the Tau effect under conditions in which the standard and comparison spatial separations and temporal intervals were separated and presented as two distinct pairs of stimulation.

Individual points of subjective equality (PSE) were computed for each of the 10 experimental conditions. Since subjects were very homogeneous in response across all conditions, the means PSEs were determined and are plotted in Figure 1 along with the standard deviations for each of the $t 1 / t 2$ ratios. The PSEs represent the mean comparison spatial separations judged to be the same as the standard separation for the different temporal relations between $t 1$ and $t 2$. Hence, the PSEs reflect the required spatial separation in the comparison stimulus pair for the Tau effect to be offset, that is, for standard and comparison spatial separations to be perceived as of equivalent extent.

Based on the raw data depicted in Figure 1, several general points can be made. (1) When $t 1$ and $t 2$ are equal, the comparison separation is judged to be nearly identical to the standard spatial separation of $10 \mathrm{~cm}$. (2) As the temporal relation between $\mathrm{t} 1$ and $\mathrm{t} 2$ becomes increasingly imbalanced, the comparison spatial separation judged to be equivalent to the standard increasingly shrinks or expands. (3) Laterality, in terms of the position of the standard and comparison stimulus pairs on either the right or left forearm, provides neither a substantial nor systematic effect.

Table 1 contains the PSEs averaged across subjects and "arms" as well as the temporal and spatial ratios (see Table note). The Tau effect is evident in columns (5) and (6); the $t 1 / t 2$ ratio is related to the reciprocal of the spatial relation, that is, to S2/S1. If the temporal and spatial ratios were perfectly reciprocally related, a condition of a true dependence of space on time would exist. Although such is not the case in the present data, the ratios $(\mathrm{t} 1 / \mathrm{t} 2: \mathrm{S} 2 / \mathrm{S} 1)$ do follow a consistent ordinal relationship, and strongly suggest that judged spatial extents, defined in terms of successive pairs of stimulation, depend more upon the relations of the temporal intervals than upon the actual spatial separations be- tween stimuli. The magnitude of the Tau effect, in terms of the percentage the comparison separation is overestimated or underestimated relative to the standard, is shown in column (7) for each of the temporal ratios; it is apparent that the spatial judgments were altered by $76 \%(49 \%+27 \%)$ by simply manipulating the temporal relations between the stimulus pairs.

In conclusion, the present results confirm and extend previous reports of the Tau effect in the tactile sense by showing that "judged spatial extents defined by successive stimulation depend more upon the temporal intervals and their relations to each other than upon the actual distances between stimuli" (Helson \& King, 1931, p. 213). As the interdependence of time and space, although highly complex, has been postulated or specified in the world of physical events (Smart, 1964) as well as in neurophysiology (Uttal, 1973), it should not be surprising to find a similar interdependence at a perceptual level.

\section{REFERENCES}

Cohen, J., Hansel, C. E. M., \& Sylvester, J. D. A new phenomenon in time judgment. Nature, 1953, 172, 901.

Cohen, J., Hansel, C. E. M., \& Sylvester, J. D. Interdependence of temporal and auditory judgments. Nature, 1954, 174, 642-645.

Cohen, J., Hansel, C. E. M., \& Sylvester, J. D. Interdependence of judgments of space, time and movement. Acta Psychologica, 1955, 11, 360-372.

GeLDREICH, E. W. A lecture room demonstration of the Tau-effect. American Journal of Psychology, 1934, 46, 483.

Helson, H., \& King, S. M. The Tau effect: An example of psychological relativity. Journal of Experimental Psychology, 1931, 14, 202-217.

Price-Williams, D. R. The Kappa effect. Nature, 1954, 173, 363.

Smart, J. J. C. Problems of space and time. New York: Macmillan, 1964.

UTtal, W. R. The psychobiology of sensory coding. New York: Harper \& Row, 1973.

Yoblick, D. A., \& SAlvendy, G. Influence of frequency on the estimation of time for auditory, visual, and tactile modalities: The Kappa effect. Journal of Experimental Psychology, 1970, 86, 157-164.

(Received for publication April 28, 1977.) 